\title{
Excitability relationships between lower limb myotatic arcs in spasticity
}

\author{
P. J. DELW A I DE, M. COR D O N N I ER, A N D M. G A D E A - C I R I A \\ From the Section of Neurology and Clinical Neurophysiology, Department of Internal Medicine, \\ University of Liege, Liege, Belgium
}

S U M M ARY The excitability of a lower limb myotatic reflex arc is modulated by the antecedent activation of another myotatic arc in the same limb; the changes can be represented by heteronymous excitability curves. This work compares heteronymous excitability curves in spastic and normal subjects. Eighteen patients who showed clear signs of pyramidal tract lesions at a chronic stage were studied. Three myotatic arcs of the lower limb (soleus, quadriceps, and short biceps) were activated either by mechanical percussion ( $T$ Sol-T Quad-TSBi) or by electrical stimulation of the afferent nerve ( $\mathrm{H}$ Sol-H Quad). They were used as conditioning and test responses in six different combinations, and the results compared to those obtained previously in normal volunteers. In spastic patients, heteronymous excitability curves are more obviously modified than homonymous ones. The changes are most prominent in the thigh muscles and less marked when the soleus reflex is conditioned by activation of quadriceps and biceps femoris myotatic reflex arcs.

Excitability curves obtained by double activation of the soleus myotatic reflex arc in normal subjects have been well known for many years (Magladery et al., 1951; Schenk, 1951; Paillard, 1955). In spasticity, the curves are modified but the changes are generally slight (Teasdall et al., 1952; Pinelli and Valle, 1960; Takamori, 1967; Yap, 1967; Zander Olsen and Diamantopoulos, 1967; Delwaide, 1971).

The excitability of a lower limb myotatic reflex arc can also be modulated by the antecedent activation of another myotatic arc in the same limb, and it is possible to represent these changes in the form of "heteronymous excitability curves" (Delwaide et al., 1976; Katz et al., 1977). Each curve has its own individual appearance, resulting from the combination of different conditioning and test myotatic arcs. In addition, certain myotatic reflex arcs (soleus and quadriceps) can be activated either by mechanical percussion of the tendon or by electrical stimulation of the afferent nerve. When the latter is used as a conditioning stimulus, the excitability curves vary as a function

Address for reprint requests: Dr P. J. Delwaide, Section of Neurology and Clinical Neurophysiology, Department of Internal Medicine, University of Liege, Liege, Belgium.

Accepted 26 January 1978 of stimulus intensity since, as the latter increases, further and more complex afferent fibres are recruited (Cordonnier and Delwaide, 1976).

The present work compares heteronymous excitability curves in spastic and normal control subjects. Our objectives were firstly to demonstrate changes which may throw some light on the physiopathology of spasticity, and secondly to compare differential reactivity in proximal and distal myotatic arcs.

\section{Patients and methods}

Eighteen patients, average age 42 years, were investigated. They all showed clear signs of fairly pure pyramidal tract disturbance in the limb under test: paresis, hypertonus of spastic type, tendon hyperreflexia (frequently with ankle clonus), and Babinski's sign; there were no other major signs of ataxia. All were in a chronic stage of their illness, able to walk with (or sometimes without) a stick. Six patients were hemiplegic, seven paraplegic, and five quadriparetic. Diagnosis included multiple sclerosis, cerebral vascular accident, and spinal cord compression.

Techniques for eliciting and recording the reflexes were the same as those described on normal subjects in an earlier publication (Delwaide et al., 
1976); the present results are compared with this normal group. Briefly, the knee and ankle of the seated subject were fixed in such a way that reflex contraction was isometric. The subject remained immobile and relaxed so as to avoid the influence exer:ed by the contraction of distant muscles. The soleus and quadriceps reflexes were activated both by afferent nerve stimulation ( $\mathrm{H}$ Sol and $\mathrm{H}$ Quad) and by tendon percussion ( $\mathrm{T}$ Sol and $\mathrm{T}$ Quad). The myotatic reflex arc of the short biceps femoris was activated by percussion of its tendon (TSBi). Electrical stimulation was used preferentially as a conditioning stimulus to avoid possible distant diffusion of the vibratory mechanical wave (Lance, 1965). When used as a test, the reflexes elicited electrically in quadriceps and soleus were adjusted to have an amplitude equal to half their maximal value $(\mathrm{H} \max / 2)$. To elicit the tendon reflexes, the tendon was percussed by an electromagnetic hammer in such a way as to obtain the largest possible responses. However, in cvery case, and especially in the spastic patients, the Jendrassik manoeuvre was efficient; this always gave a facilitation of at least $50 \%$, thus demonstrating that the reflexes had not reached saturation level.

The excitability curves have been plotted so that the reflex amplitude as a perceniage of reference value is shown on the ordinate, while the interval (in milliseconds) between conditioning and test responses is represented on the abscissa. Some combinations of conditioning and conditioned reflexes have been more frequently investigated than others (see Results); at least two different curves have been obtained for each patient. However, we have not been able to record each of the six types of interaction reported in this work for each patient, on account of the long duration of the testing procedure.

\section{Results}

CONDITIONING OF A PROXIMAL MYOTATIC REFLEX BY ACTIVATION OF A DISTAL MYOTATIC ARC

Conditioning of quadriceps tendon reflex by soleus $H$ reflex (threshold $H$ or $H$ max $/ 2$ )

In Fig. $1 \mathrm{~A}$ and $\mathrm{B}$ conditioning made by an $\mathrm{H}$ Sol
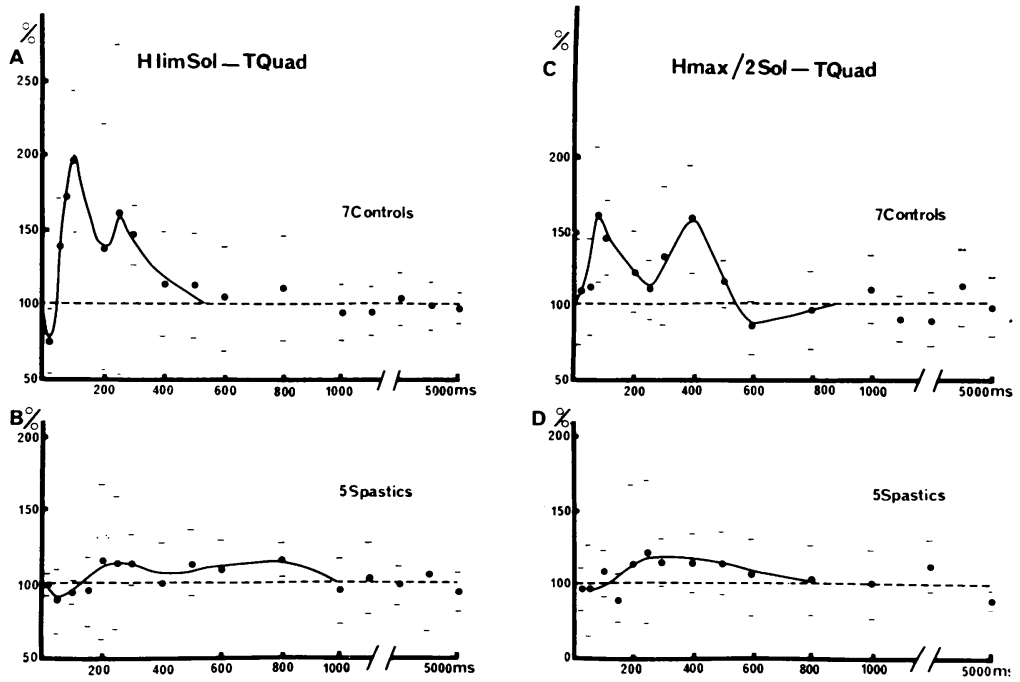

Fig. 1 Excitability curves of the quadriceps myotatic reflex arc tested by percussion of its tendon ( $T$ Quad) after conditioning by a soleus $H$ reflex ( $H \mathrm{Sol}$ ). Ordinate represents the amplitude of the reflex expressed as a percentage of unconditioned (reference) values $=100 \%$. Abscissa indicates the time interval (in milliseconds) between conditioning and test responses. $A$ and $B$ : stimulation of the medial popliteal branch of the sciatic nerve at threshold intensity for elicitation of the soleus $H$ reflex. $C$ and $D$ : as in $A$ and $B$, but nerve stimulation applied at an intensity giving rise to $a$ response of amplitude $H \max / 2 . A$ and $C$ : averaged excitability curves for seven normal subjects. $B$ and $D$ : averaged excitability curves for five spastic subjects. Dotted lines=limits of the standard deviations. 
reflex of just threshold intensity does not bring about a mechanical response which is visible. The conditioned reflex ( $\mathrm{T}$ Quad) does not reach saturation level, since it can still be facilitated by a Jendrassik manoeuvre. The control curve obtained from seven normal subjects (Fig. 1A) is compared with the mean results derived from five spastic patients (Fig. 1B). The control curve (Delwaide et al., 1976) shows first a brief inhibition (40 ms) of the conditioned reflex, followed by a strong facilitation, which lasts $600 \mathrm{~ms}$ (Fig. i A). In spastic subjects (Fig. 1B), there are slight variations from the unconditioned values: there is a weak $(10 \%)$ inhibition at the beginning, most marked at $70 \mathrm{~ms}$, followed after $200 \mathrm{~ms}$ by a moderate $(15 \%)$ facilitation lasting $200 \mathrm{~ms}$. Finally after a brief return to reference values, there is a moderate $(15 \%)$ late facilitation which lasts from the 400th to the 1000th millisecond. The standard deviations fluctuate between 6 and 25 , excluding the intervals between 200 and $300 \mathrm{~ms}$ where they reach values between 45 and 55 .

In normal subjects, the excitability curve for the quadriceps tendon reflex varies with the intensity of stimulation (as shown in Fig. 1C) of the sciatic nerve (Cordonnier and Delwaide, 1976). In spastic patients (Fig. 1D), a conditioning stimulus intensity above $\mathrm{H}$ threshold (for example, $H \max / 2$ ) makes almost no difference to the appearance of the curve in Fig. 1B, and the standard deviations of Fig. 1B and C fluctuate between the same limits.

Conditioning of electrically-induced quadriceps reflex by soleus $\mathrm{H}$ reflex $(\mathrm{H} \max / 2)$ is shown in Fig. 2 (A and B) which represents the means of curves obtained from seven normal (Fig. 2A) and six spastic subjects (Fig. 2B). In the normal subjects, after a brief inhibition $(15 \%)$ of the electrically-induced quadriceps reflex, there is a phase of facilitation (35\%) which does not last more than $200 \mathrm{~ms}$. Unlike what was shown in Fig. $1 \mathrm{~A}$ and $\mathrm{C}$, it can be seen that this facilitation is followed for several seconds by a profound inhibition (45\%). In normal subjects, there is thus a clear difference in the excitability curves according to whether the test reflex is elicited mechanically from the tendon or electrically from the nerve (Delwaide et al., in preparation).

In the spastic subjects, the electrically-induced quadriceps reflex is almost not modified. Standard deviations indicate that the small fluctuations from the base line are not significant. In spastic subjects, the curves are similar whether the quadriceps reflex is elicited mechanically from the tendon or electrically from the nerve, despite the great difference in normal subjects (compare Figs. 1B and 2B).

Conditioning of short biceps tendon reflex by soleus $H$ reflex $(H \max / 2)$

The curve derived from normal subjects (Del-
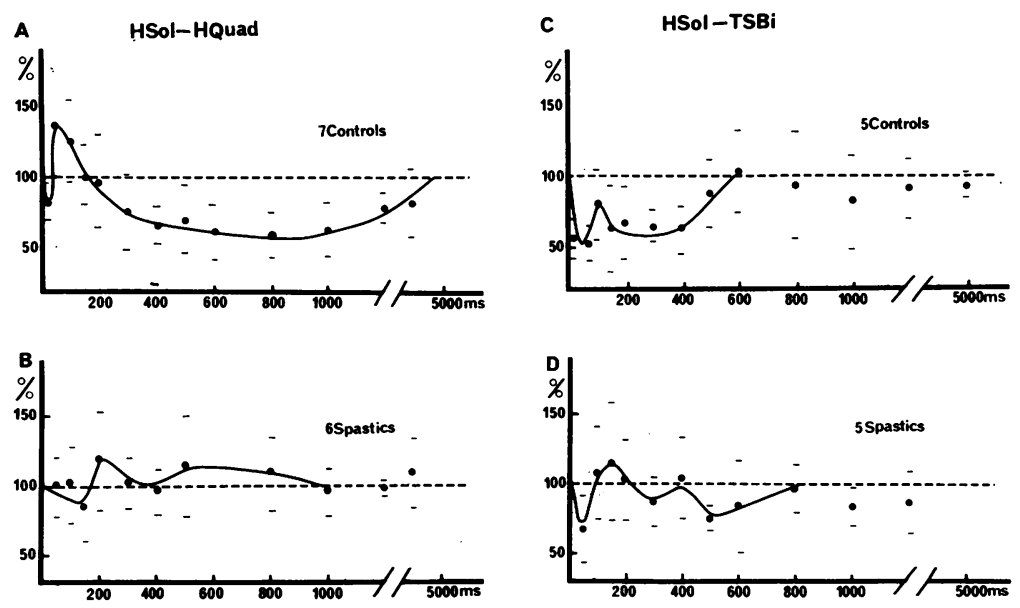

Fig. $2 A$ and B: excitability curves of the myotatic arc of the quadriceps tested by an electrically-induced reflex ( $H$ Quad) after conditioning by a soleus $H$ reflex $(H$ Sol). Stimulation of the medial popliteal branch of the sciatic nerve was applied at an intensity giving rise to a response of amplitude $\mathrm{H}$ Sol max/2. C and D: excitability curves of the reflex arc of the short biceps femoris tested by percussion of the tendon (TSBi) after conditioning by a soleus $H$ reflex ( $H$ Sol) at an intensity evoking a response at half maximal amplitude. Co-ordinates and conventions as in Fig. 1. 
waide et al., 1976) is characterised by an inhibition lasting $600 \mathrm{~ms}$ (Fig. 2C). This inhibition is of rapid onset, reaching $50 \%$ of reference value at $40 \mathrm{~ms}$. It then wanes biphasically, with a rapid phase lasting until the 90th millisecond, then a second slower recovery up to $600 \mathrm{~ms}$. As in normal subjects, the TSBi is inhibited in spastic patients during the first $100 \mathrm{~ms}$, to a value $30 \%$ below reference (Fig. 2D). This is followed by a return to unconditioned values, the test reflex is again inhibited (less than $25 \%$ below reference at $500 \mathrm{~ms}$ ) until about the 800th millisecond. It should be noted that after $800 \mathrm{~ms}$ curves obtained from spastic and normal subjects are superimposable. The standard deviations fluctuate between 10 and 35 .

CONDITIONING OF A DISTAL MYOTATIC REFLEX BY ACTIVATION OF PROXIMAL MYOTATIC ARCS

Conditioning of soleus $H$ reflex by electricallyinduced quadriceps reflex (H Quad max/2)

Figure 3 (A and B) compares the curves obtained from five normal and six spastic subjects. The normal curve (Delwaide et al., 1976) shows a moderate $(15 \%)$ and transitory inhibition of the soleus $\mathrm{H}$ reflex $(\mathrm{H} \max / 2)$ during the first $200 \mathrm{~ms}$ (Fig. 3A); it is followed by a much stronger (50\%) inhibition, characterised by a very long duration of $4000 \mathrm{~ms}$. The curve obtained from the spastic subjects (Fig. 3B) differs from the normal by a less marked $(20 \%)$ inhibition which has the same time course as in normal subjects. In spastic patients the standard deviations fluctuate between 10 and 35.

Conditioning of soleus $H$ reflex by the short biceps tendon reflex

Figure 3 (C and D) represents the curves obtained from five control (Fig. 3C) and six spastic subjects (Fig. 3D) after conditioning of the soleus $\mathrm{H}$ reflex by the tendon reflex elicited from the short biceps femoris. The normal curve shows an intense $(60 \%$ about the 100th millisecond) and prolonged (5000 ms) inhibition, marked by a slight degree of disinhibition at $200 \mathrm{~ms}$. The curve from spastic subjects is superimposable on the normal, including the rebound at $200 \mathrm{~ms}$. The standard deviations fluctuate between 5 and 30 excluding the intervals between 50 and $100 \mathrm{~ms}$ where they reach values of 40. As shown in Fig. 4, the results are reproducible when the curve is repeated in the same patient.

\section{Discussion}

The use of averaged excitability curves from pathological cases may be criticised on the grounds that spastic subjects constitute a heterogeneous population. However, such curves indicate the general characteristics of a clinical syndrome, and have been widely used in publications on double activation of the soleus myotatic reflex arc
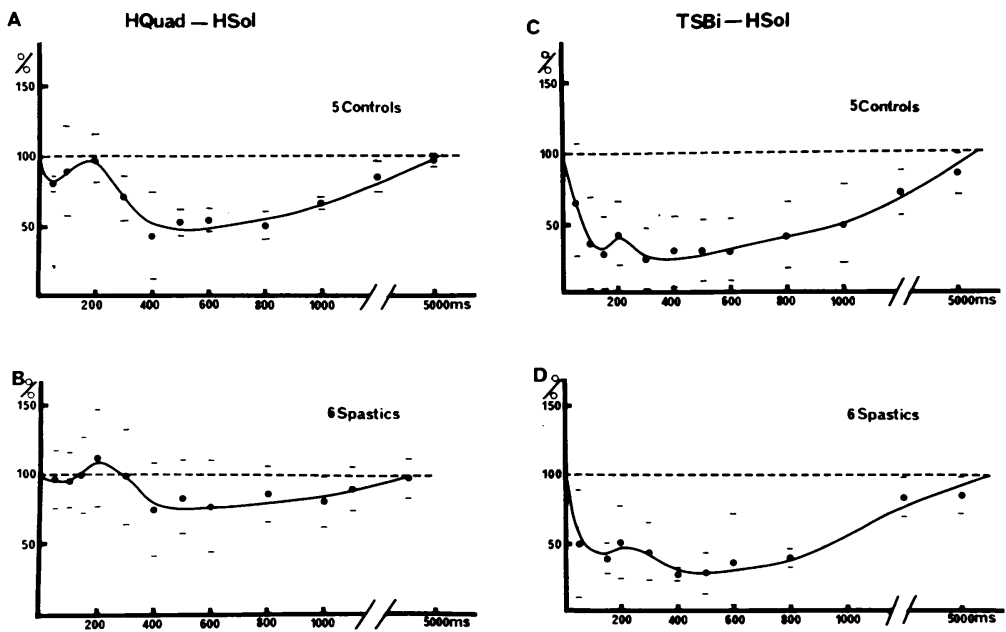

Fig. 3 Excitability curves of the soleus myotatic reflex arc tested by the $H$ reflex $(H S o l) . A$ and $B$ : conditioning by an electrically-induced quadriceps reflex (H Quad) by stimulation of the femoral nerve at an intensity giving rise to a response equal to half maximal amplitude. $C$ and $D$ : the soleus myotatic reflex arc is conditioned by the tendon reflex of the short biceps femoris (TSBi). 


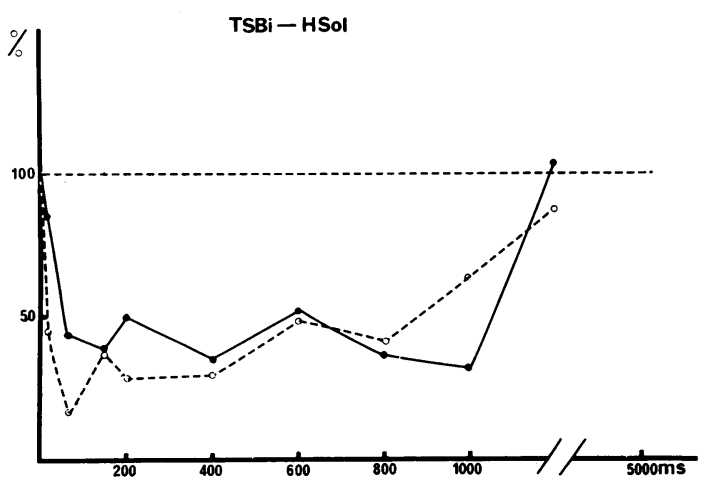

Fig. 4 Excitability curves of the myotatic reflex arc of soleus tested by $\mathrm{H}$ reflex after conditioning by $a$ short biceps tendon reflex. The two curves (continuous and interrupted lines) are individual curves performed in the same patient.

(Teasdall et al., 1952; Takamori, 1967; Yap, 1967; Zander Olsen and Diamantopoulos, 1967). Moreover, as shown in the Figures, the standard deviations are not greater in mean curves of spastic patients than in control subjects, and individual curves in spastic patients are reasonably reproducible (Fig. 4).

Mechanical percussion of quadriceps or biceps femoris tendons can be suspected, especially in spasticity, to spread to a distant muscle group and to activate their proprioceptive receptors (Lance, 1965; Katz et al., 1977). However, by using electrical stimulation of the quadriceps myotatic reflex arc, the simultaneous activation of the soleus muscle spindles by percussing the patella can be excluded.

Whether homonymous or heteronymous, the excitability curves are multiphasic, which suggests that several different mechanisms are active, each with its own time course. Without excluding a more detailed analysis of the different phases of the curves (Paillard, 1955; Delwaide et al., 1976), three periods may be considered, on the basis of latency. The first period, with a duration of approximately $80 \mathrm{~ms}$, essentially reflects purely spinal mechanisms, since the latencies for the appearance of known suprasegmental mechanisms are longer (functional stretch reflex (Melvill-Jones and Watt, 1971), transcortical reflex (Marsden et al., 1976; Nashner, 1976), spino-bulbo-spinal reflex (Shimamura et al., 1964).

The second period, of about 200 to $300 \mathrm{~ms}$, may be defined as that in which both regulatory supraspinal mechanisms and reafferentation due to the conditioning muscle contraction are concerned (Paillard, 1955; Eldred et al., 1976). This phase corresponds to the rebound of excitability seen in the homonymous $\mathbf{H}$ Sol-H Sol curves. In heteronymous curves, such as those illustrated here, this phase corresponds to a disinhibition or, in some instances, to a facilitation. Finally, a third period supervenes, characterised in general by late inhibitory phenomena. The origin of this late inhibition is unknown, but it may depend on both spinal and supraspinal mechanisms.

Because of the uncertainty about the mechanisms normally concerned in the modulation of excitability, it is not possible to give a precise neurophysiological meaning to the changes observed in spastic patients. Nevertheless they deserve some comments. Period 1 is little modified in spastic patients, in that it generally consists of inhibition, though in most instances this is less marked than in normal subjects. This suggests that short latency intersegmental mechanisms uniting the two heteronymous myotatic arcs are little changed in spastic patients. On the other hand, period 2 shows the most distinct variations from the normal pattern. This consists of quantitative modifications, such as the clear reduction in the facilitation of the quadriceps tendon reflex (Fig. 1) after a soleus $\mathbf{H}$ reflex. Period 3 shows a reduction in the normal inhibitory phenomena 2 plotted in Figs. 2C and 3A. It may, therefore, be concluded that the principal changes in the heteronymous excitability curves affect (1) the period between 80 and 300 milliseconds, and (2) period 3 (late inhibition) which is less intense.? There are, therefore, striking analogies with the changes which have been observed in the excitability curves obtained by double stimulation of the soleus myotatic arc (Teasdall et al., 1952; Zander Olsen and Diamantopoulos, 1967; Delwaide, 1971).

In normal subjects, conditioning of the quadriceps myotatic arc by the soleus $\mathrm{H}$ reflex gives rise to two very different sets of results according to whether the test reflex is elicited mechanically from the tendon or electrically from the afferent nerve (Figs. 1A and 2A). Such a difference is no longer seen in spastic subjects (Figs. 1B and 2B): however the test reflex is evoked, the conditioned curves resemble each other. It therefore makes no difference in spastic subjects, whether only the alpha-motoneurones of the quadriceps ( $\mathrm{H}$ reflex) are tested, or alpha and gamma-motoneurones simultaneously ( $T$ reflex).

Although activation of the soleus does not bring about very marked effects in proximal myotatic reflex arcs, there remains in spastic subjects some form of reciprocal innervation in the opposite modifications of excitability observed in the 
quadriceps (facilitation) and in the short biceps femoris (inhibition). Nevertheless, the antagonism seen between flexors and extensors is much less marked than in normal subjects. In spastic subjects, changes in heteronymous excitability curves are particularly evident in the thigh, compared with normal subjects. These changes are clearer than in the case of homonymous excitability curves (Schenk, 1951; Teasdall et al., 1952; Pinelli and Valle, 1960; Delwaide, 1971). It may, therefore, be useful to examine the heteronymous excitability curve in the electrophysiological investigation of spasticity. Changes from normal are, however, much less marked in the soleus reflex after activation of a proximal myotatic arc (Fig. 3). Thus there are differences in the manner in which a given pyramidal tract lesion affects the reactivity of different reflex arcs. In our experiments, these differences apparently depend more on whether the arc is proximal or distal than on whether it involves flexor or extensor muscles.

\section{References}

Cordonnier, M., and Delwaide, P. J. (1976). Conditionnement de l'arc myotatique du quadriceps par activation graduée de l'arc myotatique du soléaire. Revue Française d'Electroencephalography et Neurophysiologie Clinique, 6, 518-523.

Delwaide, P. J. (1971). Etude Expérimentale de l'Hyper-rëflexie Tendineuse en Clinique Neurologique. Editions Arscia SA: Brussels.

Delwaide, P. J., Cordonnier, M., and Charlier, M. (1976). Functional relationships between myotatic reflex arcs of the lower limb in man: investigation by excitability curves. Journal of Neurology, Neurosurgery, and Psychiatry, 39, 545-554.

Eldred, E., Hutton, R. S., and Smith, J. L. (1976). Nature of the persisting changes in afferent discharge from muscle following its contraction. In Understanding the Stretch Reflex. Progress in Brain Research. Vol. 44, p. 157. Edited by S. Homma. Elsevier Scientific Publishing Company: Amsterdam, Oxford, New York.

Katz, R., Morin, C., Pierrot-Deseilligny, E., and Hibino, R. (1977). Conditioning of $\mathbf{H}$ reflex by a preceding subthreshold tendon reflex stimulus. Journal of Neurology, Neurosurgery, and Psychiatry, 40, 475-480.

Lance, J. W. (1965). The mechanism of reflex irradiation. Proceedings of the Australian Association of Neurology, 3, 77-82.
Magladery, J. W., Teasdall, R. D., Park, A. M., and Porter, W. E. (1951). Electrophysiological studies of nerve and reflex activity in normal man. V. Excitation and inhibition of two neurone reflexes by afferent impulses in the same nerve trunk. Bulletin of the Johns Hopkins Hospital, 88, 520-537.

Marsden, C. D., Merton, P. A., and Morton, H. B. (1976). Stretch reflex and servo action in a variety of human muscles. Journal of Physiology, 259, 531560.

Melvill-Jones, G., and Watt, D. G. D. (1971). Muscular control of landing from unexpected falls in man. Journal of Physiology, 219, 729-737.

Nashner, L. M. (1976). Adapting reflexes controlling the human posture. Experimental Brain Research, 26, 59-72.

Paillard, J. (1955). Réflexes et régulations d'origine proprioceptive chez l'homme. Thèse Faculté des Sciences de Paris (Série A, no. 2858-3729), p. 293. Arnette: Paris.

Pinelli, P., and Valle, M. (1960). Studio fisiopatologico dei reflessi muscolari nelle paresi spastische (sui tests per la missura della spasticita). Archivio per le Scienze Mediche, 109, 1-127.

Schenck, E. (1951). Untersuchungen über di Hemmungsphase nach einem Zweineuronen (Eigen) reflex beim Menschen. Pflügers Archiv für die Gesamte Physiologie des Menschen und der Tiere, 253, 285-300.

Shimamura, M., Mori, S., Matsushima, S., and Fujimori, B. (1964). On the spino-bulbo-spinal reflex in dogs, monkeys and man. Japanese Journal of Physiology, 14, 411-421.

Takamori, M. (1967). H reflex study in upper motoneuron diseases. Neurology (Minneapolis), 17, 3240.

Teasdall, R. D., Park, A. M., Languth, H. W., and Magladery, J. W. (1952). Electrophysiological studies of reflex activity in patients with lesions of the nervous system. Disclosure of normally suppressed monosynaptic reflex discharge of spinal motoneurones by lesions of lower brain stem and spinal cord. Bulletin of the Johns Hopkins Hospital, 91, 245-256.

Yap, C. B. (1967). Spinal segmental and long-loop reflexes on spinal motoneurone excitability in spasticity and rigidity. Brain, 90, 887-896.

Zander Olsen, P., and Diamantopoulos, E. (1967). Excitability of spinal motoneurones in normal subjects and patients with spasticity, rigidity and cerebellar hypotonia. Journal of Neurology, Neurosurgery, and Psychiatry, 30, 325-331. 